\title{
Three Distinct Nanoviruses, One of Which Represents a New Species, Infect Faba Bean in Ethiopia
}

Adane D. Abraham, Holetta Agricultural Research Center, Ethiopian Institute of Agricultural Research, Addis Ababa, Ethiopia; Julius Kühn Institute, Federal Research Center for Cultivated Plants, 38104, Braunschweig, Germany; and Institute for Plant Pathology and Protection, University of Göttingen, 37077 Göttingen, Germany; Mark Varrelmann, Institute for Sugar Beet Research, University of Göttingen, 37079 Göttingen, Germany; and H. Josef Vetten, Julius Kühn Institute, Federal Research Center for Cultivated Plants, Germany

\begin{abstract}
Abraham, A. D., Varrelmann, M., and Vetten, H. J. 2012. Three distinct nanoviruses, one of which represents a new species, infect faba bean in Ethiopia. Plant Dis. 96:1045-1053.

In all, 70 of 296 leaf samples (23.6\%) collected from faba bean (Vicia faba) plants showing leaf yellowing and stunting in Ethiopia gave nanovirus-positive reactions when studied by triple-antibody sandwich enzyme-linked immunosorbent assay using broad-spectrum monoclonal antibodies (MAbs) specific to nanoviruses. Further analysis of these samples with seven discriminating MAbs revealed contrasting epitope profiles that were categorized into roughly three serogroups, designated A, B, and C. Serogroup A was found in $89 \%$ of the nanovirus-positive samples whereas serogroups $\mathrm{B}$ and $\mathrm{C}$ were infrequently encountered. Sequence analysis of DNA-S and DNA-U1 of

nanovirus species. Serogroup A comprised isolates of Faba bean necrotic stunt virus reported earlier only from Ethiopia and Morocco. The DNA-R, -S, -U1, and -U2 sequences of a serogroup B isolate closely resembled those of Faba bean necrotic yellows virus, providing first molecular evidence for its occurrence in Ethiopia. Sequence analysis of the eight genomic DNAs of a representative serogroup C isolate (Eth-231) showed that it shared overall nucleotide and amino acid sequence identities of only $\leq 70$ and $\leq 74 \%$, respectively, with other nanoviruses. This suggests that Eth-231 represents a new nanovirus species, for which the name faba bean yellow leaf virus is proposed.
\end{abstract} serogroup A, B, and C isolates suggested that each represents a distinct
Faba bean (Vicia faba L.) is among the major food crops grown worldwide, particularly in countries of Northeast and North Africa, Asia, and Australia. Ethiopia is the second-biggest producer of this crop after China, with production in 2008 estimated to be 688,667 metric tons (t) produced on 520,520 ha (13). In Ethiopia, however, the average faba bean yield has been consistently low $\left(<1 \mathrm{t} \mathrm{ha}^{-1}\right)$. Among the factors contributing to this low yield are plant pathogens, of which plant viruses take a significant share. Cool-season food legumes in Ethiopia have been shown to be infected by about 10 different viruses. Among those, luteoviruses and a nanovirus in faba bean and chickpea, and Pea seed-borne mosaic virus in lentil, are the most prevalent $(1-3,5,31)$, causing disease symptoms such as yellowing, leaf rolling, and plant stunting $(3,5,9)$.

Earlier observations indicated that faba bean nanovirus isolates from Ethiopia are distinct in their serological properties from those in other countries (15). Sequence analysis of the eight genomic single-stranded (ss)DNAs of a common isolate from Ethiopia substantiated this observation, indicating that it differed by over $25 \%$ in total nucleotide sequences from typical Faba bean necrotic yellows virus (FBNYV) isolates (19). In line with the current criteria for nanovirus species demarcation (33), the Ethiopian isolate was regarded as a distinct nanovirus species for which the name Faba bean necrotic stunt virus (FBNSV) has recently been proposed (19) and now awaits ratification by the International Committee of Taxonomy of Viruses (ICTV) (22).

Both FBNYV and FBNSV have a multipartite genome consisting of eight circular ssDNAs, each of which is individually encapsidated in isometric particles of about $18 \mathrm{~nm}(25,26,33)$. They

Corresponding author: H. J. Vetten,

E-mail: heinrich-josef.vetten@jki.bund.de

Accepted for publication 21 February 2012.

http://dx.doi.org/10.1094/PDIS-09-11-0734-RE

(C) 2012 The American Phytopathological Society are transmitted persistently by aphid species such as Aphis craccivora Koch. and Acyrthosiphon pisum Harris and have a host range that is largely confined to members of the family Fabaceae $(16,17)$. Although up to 12 circular ssDNAs have been found associated with nanovirus infection, eight distinct ssDNA components have been consistently identified from the isolates of all but one nanovirus species (Subterranean clover stunt virus [SCSV]), the genomes of which have been sequenced. These eight apparently integral components of the nanovirus genome are referred to as DNA-R, $-\mathrm{S},-\mathrm{C},-\mathrm{M}$, and $-\mathrm{N}$, which code for master replication initiator (M-Rep), structural (capsid), cell-cycle link, movement, and nuclear shuttle proteins, respectively, and as DNA-U1, -U2, and -U4, which encode proteins of as yet unknown functions (33). In contrast, any other DNAs are not consistently associated with nanovirus infections and, thus, considered satellite-like DNAs, which encode a Rep protein capable of initiating only autonomous replication (33). Recently, the eight cloned DNAs of FBNSV have been used to reconstitute a fully infectious and aphid-transmissible nanovirus with symptoms comparable with those of a wild-type virus in the field, confirming that they do, indeed, form the entire nanovirus genome (19).

Control of nanovirus aphid vectors using insecticides is both costly and unreliable; therefore, a safer and more sustainable alternative is the use of resistant varieties $(27,28)$. Information on virus diversity or variability in a geographic area is important for selection of plant genotypes in breeding programs with resistance traits against the whole range of natural variants. Initial information on nanoviruses species infecting faba bean suggested that their geographic distribution areas do not overlap, with FBNYV occurring chiefly only in West Asia and North Africa, FBNSV in Ethiopia, Milk vetch dwarf virus (MDV) in East Asia, and SCSV in Australia (33). However, this notion has recently been questioned by the finding that different but closely related nanoviruses species, FBNYV and FBNSV, were found in the same area in Morocco (4) Despite the information that nanovirus isolates in Ethiopia are serologically and genetically distinct from FBNYV $(15,18)$, most previous reports on the occurrence and relative importance of na- 
noviruses for legume crops in Ethiopia refer only to FBNYV and made no attempt to discriminate nanovirus isolates $(3,31)$. This prompted us to study a larger number of faba bean samples from Ethiopia using a wider range of differentiating monoclonal antibodies (MAbs) and sequence analysis. The data obtained indicate that nanoviruses infecting faba bean in Ethiopia belong to three related but distinct species, with FBNSV being the predominant one and both FBNYV and a previously unrecognized nanovirus species being apparently of minor significance. An isolate of the latter species is characterized here by describing the complete sequence of its eight genomic ssDNAs.

\section{Materials and Methods}

Sample collection and serological analysis. Leaf samples collected in a field survey conducted in the major faba-bean-growing areas of Ethiopia during the main growing season of 2002 were used in this study (Fig. 1). On each survey route, fields were selected along the main roads and plants showing symptoms suggestive of nanovirus infection such as yellowing, stunting, necrosis, and leaf deformation were collected and preserved by drying over $\mathrm{CaCl}_{2}$. When there were no visible virus symptoms, leaves from about five apparently healthy plants were randomly collected as a composite sample for subsequent serological analysis. In total, 112 fields were visited and 296 samples were collected and analyzed serologically by triple-antibody sandwich enzyme-linked immunosorbent assay (TAS-ELISA) (15) using polyclonal immunoglobulin $\mathrm{G}$ to FBNYV for trapping of nanovirus antigen (26). Following incubation of leaf extracts, plates were incubated with appropriate dilutions of (i) a broad-spectrum MAb mixture (1-1F2, 2-1A1, and 3-4F2) thought to react with all known nanoviruses such as FBNYV, FBNSV, MDV, and SCSV (15); (ii) three differentiating MAbs (1-3D8, 2-3E12-D5, and 3-4A5) raised against an Egyptian isolate of FBNYV (FBNYV-Eg) (15); and (iii) four MAbs (82G10, 8-3G11, 8-4F9, and 8-6F8-A5) specific to an Ethiopian isolate of FBNSV (FBNSV-Eth) (19). Nanovirus-positive samples that gave an epitope profile similar to that of FBNSV-Eth and
FBNYV-Eg were assigned to serotype A and B, respectively, whereas serotype $\mathrm{C}$ comprised isolates that failed to react with all FBNSV-Eth MAbs and gave strong and weak reactions with the FBNYV-Eg MAbs 1-3D8 and 3-4A5, respectively. The major difference between serotype $\mathrm{B}$ and $\mathrm{C}$ was the strength of the reaction with MAb 3-4A5.

Oligonucleotide primers and immunocapture polymerase chain reaction. To amplify a part of one or more of the non-Rep DNA components of the isolates Eth-2, Eth-218, and Eth-231, two primer pairs (P23/P44 and P75/P76) designed from conserved sequences in noncoding regions in previous works (19) were used in polymerase chain reaction (PCR). DNA-R of Eth-2, Eth-218, and Eth-231 was specifically amplified by primer pair P3/P20 (32). After restriction fragment length polymorphism-based selection of specific DNA components and sequencing as described previously (4), additional primer pairs specific to each DNA component were designed and used for selective amplification or to obtain the complete sequence of an already identified component. Further primer pairs used for completing the sequence of all eight DNAs of Eth-231 are described in Table 1. Amplification of viral sequences was done using an immunocapture PCR procedure described previously (4).

Purification and cloning of PCR products were performed using standard protocols essentially as described (4). DNA sequencing was carried out by a commercial company (MWG Biotech, Ebersberg, Germany). Unless otherwise stated, a minimum of two overlapping clones, sequenced in both directions, were used to generate the sequences of the individual components. Sequence assembly and pairwise comparison were performed using the DNAMAN program (Lynnon Biosoft, Quebec, Canada). Database search for related sequences was carried using the BLAST program (6). Phylogenetic trees were constructed using the neighbor-joining algorithm included in the DNAMAN software package. GenBank accession numbers of representative nanovirus sequences used for sequence comparison or phylogenetic analysis were as follows: Egyptian isolate of FBNYV (FBNYV-[EG]; AJ132179 to AJ132184, AJ132186, and AJ749902), Syrian isolate of FBNYV

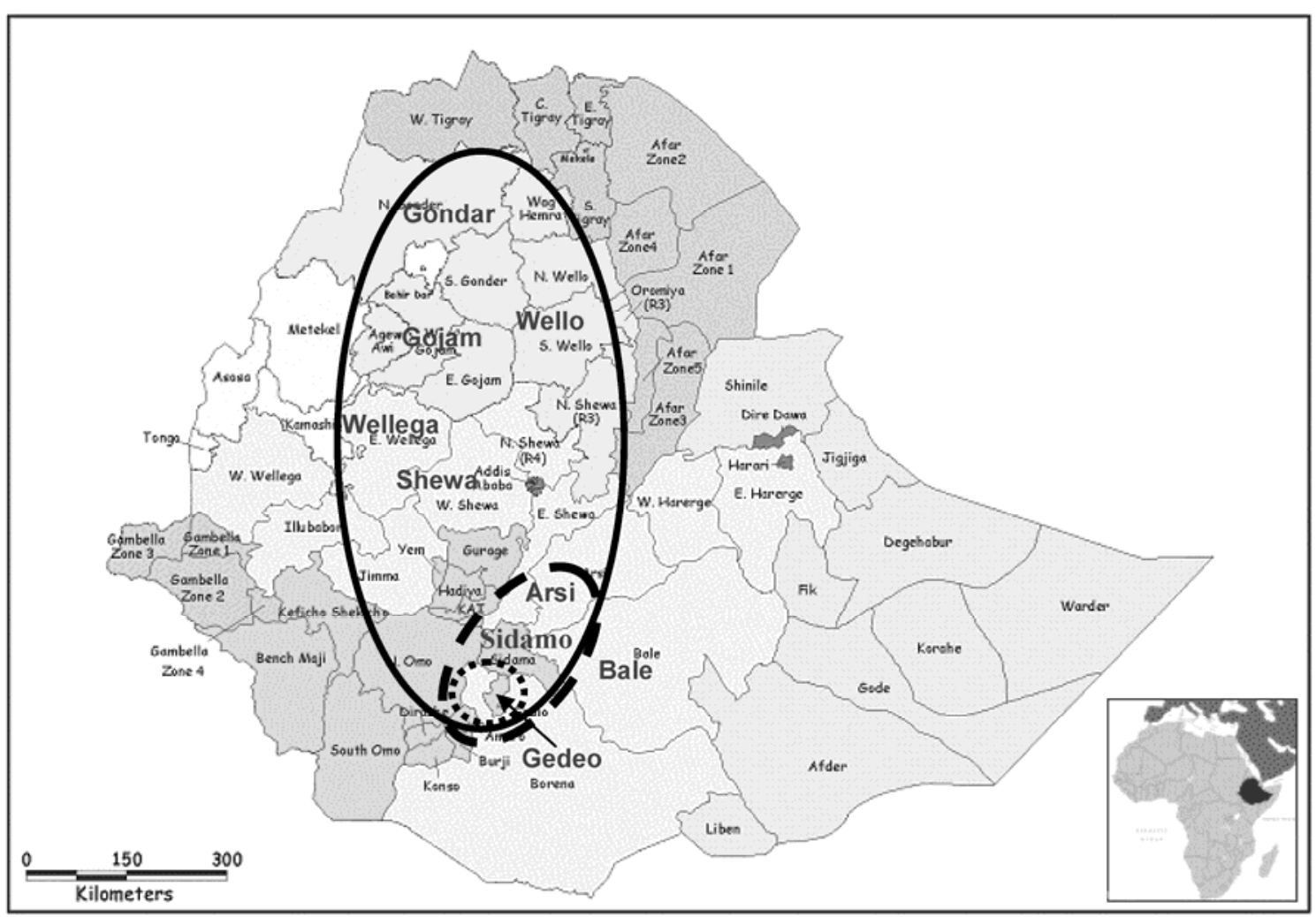

Fig. 1. Map of Ethiopia showing the areas covered in the survey for sample collection and the distribution of the different nanovirus serogroups. Areas where the different serogroups occur are encircled with a solid line for serogroup $A$, a dashed line for serogroup $B$, and a dotted line for serogroup $C$ isolates. 
(FBNYV-[SY]; Y11405 to Y11409, AJ005965, AJ005967, and AJ749903), Moroccan isolate of FBNYV (FBNSV-MA; GQ274023 to GQ274030), Ethiopian isolate of FBNSV (FBNSV[ET]; GQ150778 to GQ150784), Moroccan isolate of FBNSV (FBNSV-MA; GQ274031 to GQ274038), Japanese isolate of MDV (MDV-[JP]; AB000923 to AB000927, AB009046, AB027511, and AB255373), Australian isolates of SCSV (SCSV-[AU]; AJ290434 and U16730, -2, -3, -4, and -6); and Banana bunchy top virus (BBTV-[AU]; S56276 and L41574 to -78). The latter were used as out-group sequences.

\section{Results}

Distribution and variation in epitope profile of faba bean nanovirus isolates. Of the total of 296 samples collected from 112 faba bean fields throughout the country (Fig. 1) and analyzed serologically, $70(23.6 \%)$ were infected by nanoviruses as determined by the reaction with the broad-spectrum MAbs (Table 2). The geographic distribution of the nanovirus-infected samples collected and detected in different areas is presented in Table 2.

Serotyping of the 70 nanovirus-positive samples from Ethiopia using the seven discriminating MAbs revealed the occurrence of isolates differing in epitope profiles. They were categorized into three groups as represented by the samples Eth-2, Eth-218, and Eth-231 (Table 3). The vast majority $(88.6 \%)$ of the infected samples (e.g., Eth-2) produced epitope profiles similar to that of FBNSV-Eth and, for the purpose of this article, is referred to here as serogroup A (Table 3). Serogroup A isolates occurred in all areas surveyed (Table 2; Fig. 1) and were the only type found in samples from northern (Gojam, Gonder, and Wello) and central (Shewa and Wellega) Ethiopia, the main faba-bean-growing regions.
Some of the samples originating from southeastern (Tiyo area in Arsi region) and southern (Gedeo area in Sidamo region) Ethiopia (Fig. 1) produced epitope profiles distinct from those of the serogroup A isolates. Some isolates represented by Eth-218 (Table 3) reacted strongly with MAbs $1-3 \mathrm{D} 8$ and 3-4A5 specific to FBNYV isolates (15) but not with three of the four MAbs to FBNSV-Eth. Ethiopian isolates with such an epitope profile have not been reported before and are designated serogroup B. This group appeared to comprise FBNYV isolates previously reported from nearly all countries of West Asia and North Africa $(15,26)$. A third category (serogroup C), consisting of four isolates that originated only from the Gedeo area, southern Ethiopia, reacted strongly with MAb 1-3D8 and weakly with MAb 3-4A5 but failed to react with any MAbs to FBNSV-Eth. Because such an epitope profile has not previously been observed for any nanovirus isolate, they appeared to be novel isolates represented by isolate Eth-231 (Fig. 1).

Sequence analysis of serologically distinct nanovirus isolates. The nanovirus-positive samples Eth-2, Eth-218, and Eth-231 representing serogroups $\mathrm{A}, \mathrm{B}$, and $\mathrm{C}$, respectively (Table 3), were selected for sequence analysis. The types of sequence information generated here for the two former isolates were as follows. DNA-S and -U1 of Eth-2 and DNA-S of Eth-218 were only partially sequenced, with the sequenced part encompassing the coding region. Complete sequences were obtained for the DNA-R (1,003 nucleotides [nt]), -U1 (990 nt), and -U2 (988 nt) of Eth-218 (Table 4). For Eth-231, all eight genomic DNAs were completely sequenced (Table 5). The sequences of the four and eight DNA components of Eth-218 and Eth-231, respectively, have been deposited in the European Nucleotide Archive (accession numbers HE663168 to HE663171 and HE654123 to HE654130, respectively).

Table 1. Additional primer pairs used for specific amplification of the eight DNA sequences of the Ethiopian nanovirus isolate Eth-231

\begin{tabular}{|c|c|c|}
\hline Genome component & Primer name and orientation ${ }^{\mathrm{a}}$ & Primer sequence $\left(5^{\prime} \rightarrow 3^{\prime}\right)$ \\
\hline \multirow{2}{*}{ DNA-M } & PLo F & TAGTATTACCCCCGCCC \\
\hline & PEt4 R & СССААТTCTTТСТСТСТСА \\
\hline \multirow[t]{2}{*}{ DNA-U1 } & PLo F & TAGTATTACCCCCGCCC \\
\hline & PEt3 R & GTGTAAACTTTCATACGACA \\
\hline \multirow[t]{2}{*}{ DNA-N } & Et8 F & ATGGCAGATTGGGTTTTCTAGTC \\
\hline & Et8 R & CACTTTGATTCTGAGTGAATG \\
\hline \multirow{2}{*}{ DNA-U4 } & Et12 F & CCСTTTGAAGATGCAGATCCA \\
\hline & Et12 R & TTTCATCTGCACAATCCACA \\
\hline \multirow[t]{2}{*}{ DNA-S } & Et5 F & GATCTTGGTACTCTGCGAAGCA \\
\hline & Et5 R & CTTAGTACAATCTGTCCCAGCAA \\
\hline \multirow[t]{2}{*}{ DNA-C } & Et10 F & ACATATGAAGCTCTCTGCATA \\
\hline & Et10 R & CATATTTAACTCTGCAGCAGGGTA \\
\hline \multirow[t]{2}{*}{ DNA-U2 } & $\mathrm{P} 50 \mathrm{~F}$ & TTAAGTTAATAAATGACATCTATC \\
\hline & P55 R & GCTGATACTATCCTTCCATG \\
\hline
\end{tabular}

a Primer orientation: sense (F) and anti-sense (R).

Table 2. Geographic origin of the faba bean samples from Ethiopia and their assignment to the different nanovirus serogroups on the basis of their reactions with the seven monoclonal antibodies (MAbs)

\begin{tabular}{|c|c|c|c|c|c|c|}
\hline \multirow[b]{2}{*}{ Regions } & \multicolumn{3}{|c|}{ Number of } & \multicolumn{3}{|c|}{ Number of samples belonging to serogroups ${ }^{a}$} \\
\hline & Fields surveyed & Samples tested & Positive samples $^{b}$ & $\mathbf{A}$ & B & $\mathbf{C}$ \\
\hline Addis Ababa & 3 & 6 & 2 & 2 & - & - \\
\hline Arsi & 11 & 12 & 6 & 4 & 2 & - \\
\hline Bale & 10 & 10 & 2 & 2 & - & - \\
\hline Gonder & 26 & 101 & 10 & 10 & - & - \\
\hline Gojam & 11 & 44 & 5 & 5 & - & - \\
\hline Shewa & 19 & 34 & 14 & 14 & - & - \\
\hline Sidamo (Gedeo) & 8 & 12 & 7 & 1 & 2 & 4 \\
\hline Wellega & 6 & 9 & 6 & 6 & - & - \\
\hline Wello & 18 & 68 & 18 & 18 & - & - \\
\hline Total & 112 & 296 & 70 & 62 & 4 & 4 \\
\hline
\end{tabular}

a $\mathrm{A}=$ nanovirus samples that reacted with all Faba bean necrotic stunt virus (FBNSV)-Eth-specific MAbs (8-2G10, 8-3G11, 8-4F9, and 8-6F8-A5), B = nanovirus samples that reacted only with two (1-3D8 and 3-4A5) of the three differentiating MAbs raised against Faba bean necrotic yellows virus (FBNYV)-Eg, and $\mathrm{C}=$ samples that failed to react with all FBNSV-Eth MAbs and gave strong and weak reactions with the FBNYV-Eg MAbs 1-3D8 and 3-4A5, respectively.

b Nanovirus-positive samples. Samples that reacted with a broad-spectrum monoclonal antibody mixture (1-1F2, 2-1A1, and 3-4F2) were considered infected with a nanovirus. 
Because DNA-S and -U1 encode well- and less-conserved nanovirus proteins, respectively (33), they were identified and sequenced from randomly selected clones of all three isolates. The amino acid sequences of the proteins potentially encoded by these DNAs were used to assess the genetic diversity and phylogenetic relationship of these nanovirus isolates to each other and to representative isolates of other nanovirus species. The amino acid sequence identities obtained by pairwise comparison of the capsid protein $(\mathrm{CP})$ and $\mathrm{U} 1$ gene sequences of these isolates with those of previously sequenced nanovirus isolates revealed that there is a close correlation between the serogroup assignment and sequence identity of the isolates (Table 4). For example, Eth-2 was almost indistinguishable (approximately 99\%) from FBNSV-Eth (GQ150779 and GQ150783) in both CP and U1 protein amino acid sequence identities, indicating that they belong to the same species
(Table 4). On the other hand, isolate Eth-218 shared CP and U1 protein amino acid sequence identities of 96 and $93 \%$, respectively, with FBNYV-Eg, suggesting that they are isolates of the same virus species (FBNYV). This contrasts to the significantly lower identities obtained when Eth-2 or FBNSV-Eth is compared with Eth-218 or FBNYV-Eg, which gave identity values of less than $85 \%$ for $\mathrm{CP}$ and $68 \%$ for $\mathrm{U} 1$ protein amino acid sequences (Table 4). In line with this result, the phylogenetic analysis of proteins encoded by four DNAs of Eth-218 (DNA-S, -R, -U1 and -U2) confirmed the assignment of Eth-218 to FBNYV (Fig. 2).

Genome sequence of the Eth-231 isolate and its relationship to other nanoviruses. Because analysis of the $\mathrm{CP}$ and $\mathrm{U} 1$ protein amino acid sequences of Eth-231 (Table 4) suggested that it might represent a novel nanovirus species, its eight genomic DNAs were completely amplified, cloned, sequenced, and analyzed. The eight

Table 3. Nanovirus-positive samples from Ethiopia producing distinct epitope profiles and representing the three serogroups on the basis of TAS-ELISA reactions with seven discriminating monoclonal antibodies (MAbs) ${ }^{\mathrm{a}}$

\begin{tabular}{|c|c|c|c|c|c|c|c|c|c|}
\hline \multirow[b]{3}{*}{ Samples $^{\text {b }}$} & \multirow[b]{3}{*}{ MAb mixture } & \multicolumn{7}{|c|}{ Differentiating MAbs raised against } & \multirow[b]{3}{*}{ Serogroup $^{c}$} \\
\hline & & \multicolumn{4}{|c|}{ FBNSV-Eth } & \multicolumn{3}{|c|}{ FBNYV-Eg } & \\
\hline & & 8-2G10 & 8-3G11 & $8-4 F^{9}$ & 8-6F8-A5 & 1-3D8 & $3-4 A 5$ & 2-3E12-D5 & \\
\hline Eth-2 & +++ & +++ & +++ & +++ & +++ & - & - & - & A \\
\hline Eth-218 & +++ & - & - & - & - & ++ & +++ & - & B \\
\hline Eth-231 & +++ & - & - & - & - & +++ & + & - & $\mathrm{C}$ \\
\hline FBNSV-Eth & +++ & +++ & +++ & +++ & +++ & - & - & - & A \\
\hline FBNYV-Eg & +++ & - & - & - & - & +++ & ++ & +++ & B \\
\hline
\end{tabular}

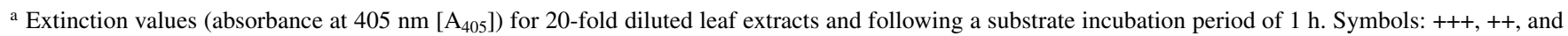
+ indicate $\mathrm{A}_{405}$ values $<0.8$, between 0.2 and 0.8 , and $<0.2$, respectively; - indicates absorbance values less than two times the value of a noninfected control.

b Representative samples. Faba bean necrotic stunt virus Egypt (FBNYV-Eg) and Faba bean necrotic stunt virus Ethiopia (FBNSV-Eth) against which the monoclonal antibodies had been raised were used as control antigen.

c $\mathrm{A}=$ nanovirus samples that reacted with all FBNSV-Eth-specific MAbs $(8-2 \mathrm{G} 10,8-3 \mathrm{G} 11,8-4 \mathrm{~F} 9$, and 8-6F8-A5), B = nanovirus samples that reacted only with two (1-3D8 and 3-4A5) of the three differentiating MAbs raised against FBNYV-Eg, and C= samples that failed to react with all FBNSV-Eth MAbs and gave strong and weak reactions with the FBNYV-Eg MAbs 1-3D8 and 3-4A5, respectively.

Table 4. Pairwise comparison of predicted capsid protein (below diagonal) and U1 (above diagonal) amino acid sequences of the three Ethiopian isolates (Eth-2, -218, and -231) with representative isolates of Faba bean necrotic stunt virus (FBNSV) from Holetta, Ethiopia (Eth), Faba bean necrotic yellows virus (FBNYV) from Egypt (Eg), Milk vetch dwarf virus (MDV), and Subterranean clover stunt virus (SCSV)

\begin{tabular}{|c|c|c|c|c|c|c|c|}
\hline \multirow[b]{2}{*}{ Viruses, isolates } & \multicolumn{3}{|c|}{ Ethiopian isolates studied here } & \multirow[b]{2}{*}{ FBNSV-Eth } & \multirow[b]{2}{*}{ FBNYV-Eg } & \multirow[b]{2}{*}{ MDV } & \multirow[b]{2}{*}{ SCSV } \\
\hline & Eth-2 & Eth-218 & Eth-231 & & & & \\
\hline Eth-2 & $\ldots$ & 65.8 & 66.4 & 99.4 & 64.5 & 68.7 & 44.1 \\
\hline Eth-218 & 84.9 & $\ldots$ & 68.2 & 66.4 & 92.9 & 75.3 & 44.8 \\
\hline Eth-231 & 88.4 & 89.0 & $\ldots$ & 67.3 & 65.6 & 71.3 & 42.4 \\
\hline FBNSV-Eth & 98.8 & 84.7 & 87.2 & $\ldots$ & 65.1 & 69.3 & 44.8 \\
\hline FBNYV-Eg & 84.3 & 95.9 & 88.4 & 83.7 & $\ldots$ & 74.0 & 42.1 \\
\hline MDV & 83.7 & 83.7 & 82.6 & 83.7 & 83.7 & $\ldots$ & 46.5 \\
\hline SCSV & 56.2 & 57.1 & 56.7 & 55.6 & 55.6 & 53.6 & $\ldots$ \\
\hline
\end{tabular}

Table 5. Sizes of the eight single-stranded DNAs, their encoded proteins, positions, and sequence of some transcription signals of the Eth-231 isolate

\begin{tabular}{|c|c|c|c|c|c|c|c|c|}
\hline \multirow[b]{2}{*}{$\begin{array}{l}\text { DNA } \\
\text { component }^{b}\end{array}$} & \multirow[b]{2}{*}{$\begin{array}{l}\text { Size } \\
(\mathbf{n t})^{\mathrm{c}}\end{array}$} & \multirow[b]{2}{*}{$\begin{array}{c}\text { TATA-box } \\
\text { positiond }^{d}\end{array}$} & \multicolumn{4}{|c|}{ ORF (predicted) ${ }^{\mathrm{a}}$} & \multirow[b]{2}{*}{$\begin{array}{l}\text { Termination } \\
\text { codon }\end{array}$} & \multirow[b]{2}{*}{$\begin{array}{c}\text { Polyadenylation } \\
\text { signal }^{\text {d }}\end{array}$} \\
\hline & & & $\begin{array}{c}\text { Initiation } \\
\text { position }^{e}\end{array}$ & $\begin{array}{l}\text { Codon, } \\
\text { context }^{\text {f }}\end{array}$ & $\begin{array}{l}\text { Number of } \\
\text { amino acids }\end{array}$ & $\begin{array}{l}\text { Protein size } \\
\quad(k D a)\end{array}$ & & \\
\hline $\mathrm{R}$ & 1,002 & 54 & 100 & AATATGG & 286 & 33.1 & TGA & 92 \\
\hline S & 1,001 & 258 & 296 & AAAATGG & 183 & 20.5 & TAA & 842 \\
\hline M & 980 & 277 & 333 & CGAATGG & 112 & 12.7 & TAG & 230 \\
\hline $\mathrm{C}$ & 995 & 263 & 303 & AAAATGG & 173 & 20.1 & TGA & 842 \\
\hline $\mathrm{N}$ & 1,000 & 279 & 321 & AAAATGG & 153 & 17.3 & TAA & 58 \\
\hline U1 & 990 & 265 & 356 & TTCATGG & 154 & 17.9 & TAA & 823 \\
\hline $\mathrm{U} 2$ & 995 & 277 & 346 & CTTATGG & 121 & 14.8 & TAA & 806 \\
\hline U4 & 972 & 242 & 340 & TTTATGG & 106 & 12.2 & TAA & 828 \\
\hline
\end{tabular}

a $\mathrm{ORF}=$ open reading frame.

b Sequences of these DNA components have been assigned the European Nucleotide Archive accession number HE654123 to HE654130.

c Size in nucleotides (nt).

${ }^{\mathrm{d}}$ Position of potential TATA box and polyadenylation signal is the number of nucleotides from the first nucleotide upstream of the replication origin to the first base of the respective motif.

e Position of the ORF is the number of bases from the first base $3^{\prime}$ of the replication origin and includes the first base of the initiation codon.

f Initiation codon and its context. 


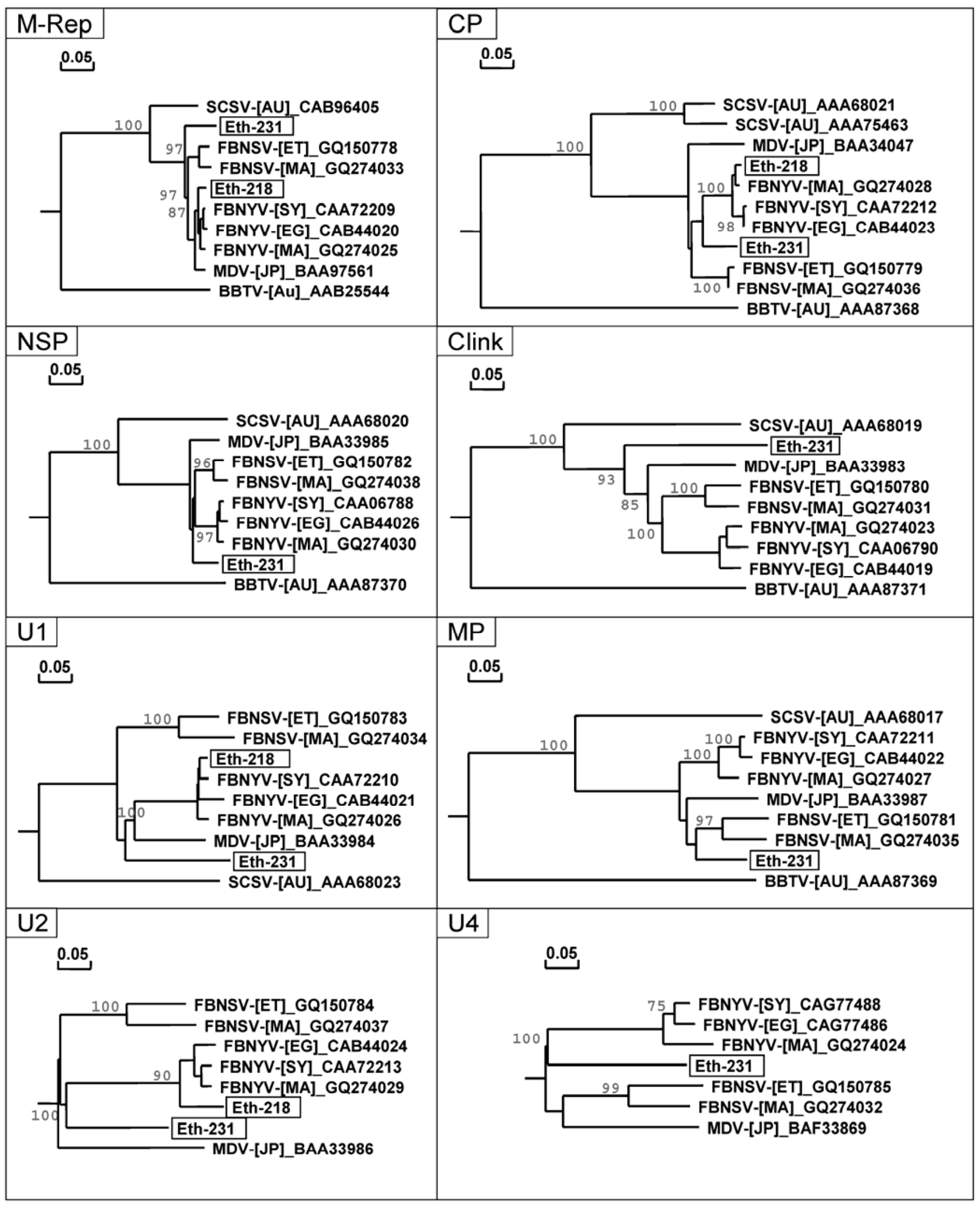

Fig. 2. Neighbor-joining dendrograms illustrating the amino acid sequence relationships in the master replication (M-Rep), capsid (CP), cell-cycle link (Clink), movement (MP), nuclear shuttle (NSP), U1, U2, and U4 proteins encoded by the eight DNAs of Eth-231 and, in the M-Rep, CP, U1, and U2 proteins encoded by the four DNAs of Eth218 with the homologous proteins of Banana bunchy top virus (BBTV), Faba bean necrotic yellows virus (FBNYV), Faba bean necrotic stunt virus (FBNSV), Milk vetch dwarf virus (MDV), and Subterranean clover stunt virus (SCSV). Because sequence information is available for genetically distinct isolates of some nanovirus species, the sequences of FBNYV isolates from Egypt (EG), Syria (SY), and Morocco (MA) and FBNSV isolates from Ethiopia (ET) and Morocco (MA) were included in the comparison. Vertical branch lengths are arbitrary and horizontal distances are proportional to the number of base substitutions per site (see scale bar). Sequence alignments and dendrograms were produced using DNAMAN (version 6, Lynnon Biosoft, Quebec, Canada) which uses a CLUSTAL-type algorithm. Dendrograms were bootstrapped 1,000 times (scores higher than $75 \%$ are shown at nodes). 
ssDNAs forming its genome ranged in size from $972 \mathrm{nt}$ (DNA-U4) to 1,002 nt (DNA-R), amounting to a total genome size of 7,935 nt (Table 5). All eight DNAs appeared to be structurally similar in having a common stem loop sequence and one major open reading frame (ORF) that potentially encodes one of the eight distinct nanovirus proteins. Comparison of the total nucleotide sequence of Eth-231 with those of other nanoviruses revealed overall nucleotide sequence identities of $64.4 \%$ (SCSV) to $72.8 \%$ (MDV) (Table 6).

Analysis of the noncoding regions of the Eth-231 DNAs. The noncoding region (NCR) of each of the Eth-231 DNAs has a highly conserved stretch of 33 or 35 bases capable of forming a putative stem-loop structure, as has been already reported for other nanoviruses $(11,25,30,32)$. The nonanucleotide sequence TAGTATTAC forming the loop and shown to include the origin of virus replication (32) is perfectly conserved in all genomic DNAs. The stem loop sequence of all but two DNAs of Eth-231 starts with nucleotides CT followed by a GC-rich sequence and ends with a matching AG (Fig. 3), while DNA-N and -S have an additional $\mathrm{C}$ at the beginning and a matching $G$ at the end. In addition, these two DNAs have a stem sequence that differs in four bases, TG at positions 8 and 9 and the matching nucleotides $\mathrm{AC}$ at the other side of the stem loop (Fig. 3A). Here, Eth-231 differs from other nanoviruses, such as FBNYV (25), FBNSV (19), and MDV (30), which have almost perfectly conserved stem-loop sequences differing only in stem lengths ( \pm CT-AG; 32). In addition, the common sequence motifs flanking the stem loop, which are proposed to act as binding sites for the M-Rep protein (32), are also somewhat different in DNA-N and $-\mathrm{S}$ as compared with those of FBNYV, FBNSV, and MDV (Fig. 3A). Moreover, the AatII site (GACGTC) universally conserved in the common region of most nanoviruses is absent in DNA-U1, -N, and -S of Eth-231. The common region II in the NCR (a coding sequence in DNA-R) has perfectly conserved domains (Fig. 3B) where endogenous primers may bind during self priming to generate the complementary strand DNA synthesis for infection, as was demonstrated for BBTV by Hafner et al. (20).

The NCRs of DNA-U1 and $-\mathrm{C}$ as well as those of DNA-M and -U2 share identities of 93\% (Fig. 4). The NCR of DNA-N is most distinct from that of all the other DNAs. The NCRs of DNA-S and -U4 are more divergent from each other although they share a higher identity with DNA-C and U1. Because of the large ORF of DNA-R, this component has a very small NCR flanking the stem loop sequence and, thus, was not included in the comparison. Similar relationships have previously been described for other nanovirus isolates $(4,19,21,24,30)$.

For each DNA, the total nucleotide size, the properties of the ORFs (i.e., position and context of initiation codon, the number of amino acids, and the stop codons) are presented in Table 5. The potential TATA-box sequence (TATAAA) is conserved in all DNAs and located at a distance of $38 \mathrm{nt}$ (DNA-S) to $98 \mathrm{nt}$ (DNA-U4) from the initiation codon (Table 5). A possible polyadenylation

Table 6. Total nucleotide and amino acid (coding) sequence identities between homologous DNAs of Eth-231 and other members of the family Nanoviridae

\begin{tabular}{|c|c|c|c|c|c|c|c|c|c|c|c|c|c|c|c|c|c|c|}
\hline \multirow[b]{3}{*}{ Virus $^{b}$} & \multicolumn{18}{|c|}{ DNA components ${ }^{a}$} \\
\hline & \multicolumn{2}{|c|}{$\mathbf{R}$} & \multicolumn{2}{|c|}{$\mathbf{S}$} & \multicolumn{2}{|c|}{$\mathbf{M}$} & \multicolumn{2}{|c|}{$\mathbf{C}$} & \multicolumn{2}{|c|}{$\mathbf{N}$} & \multicolumn{2}{|c|}{ U1 } & \multicolumn{2}{|c|}{ U2 } & \multicolumn{2}{|c|}{ U4 } & \multicolumn{2}{|c|}{ Mean } \\
\hline & nt & $\mathbf{a a}$ & nt & $\mathbf{a a}$ & nt & $\mathbf{a a}$ & nt & $\mathbf{a a}$ & nt & $\mathbf{a a}$ & nt & aa & nt & $\mathbf{a a}$ & nt & aa & nt & $\mathbf{a a}$ \\
\hline FBNSV-Eth & 87.5 & 90.9 & 75.3 & 87.2 & 68.4 & 81.3 & 70.0 & 60.4 & 75.8 & 91.5 & 70.0 & 65.6 & 60.1 & 65.3 & 62.4 & 54.7 & 70.4 & 74.6 \\
\hline FBNYV-Eg & 88.2 & 90.9 & 78.0 & 88.4 & 64.8 & 80.4 & 70.3 & 58.0 & 75.8 & 89.5 & 69.0 & 65.6 & 58.6 & 61.2 & 57.4 & 55.2 & 70.4 & 73.6 \\
\hline MDV-Jp & 86.6 & 92.0 & 78.4 & 82.6 & 65.3 & 79.5 & 73.8 & 59.2 & 76.5 & 90.8 & 71.3 & 68.7 & 69.7 & 60.3 & 60.8 & 50.9 & 72.8 & 73.0 \\
\hline SCSV-Au & 77.7 & 82.2 & 63.8 & 56.7 & 62.5 & 45.0 & 59.5 & 40.5 & 63.9 & 68.0 & 58.8 & 37.0 & - & - & - & - & 64.4 & 54.9 \\
\hline BBTV-Au & 56.9 & 53.1 & 48.4 & 17.4 & 49.7 & 17.6 & 52.7 & 17.0 & 55.5 & 44.1 & - & - & - & - & - & - & $\ldots$ & $\ldots$ \\
\hline
\end{tabular}

${ }^{a}$ Nucleotides (nt) and amino acids (aa); - indicates that a comparison was not possible due to the fact that a homologous DNA component has not yet been identified for the other nanovirus.

${ }^{\mathrm{b}}$ Faba bean necrotic stunt virus (FBNSV) from Holetta, Ethiopia (Eth); Faba bean necrotic yellows virus (FBNYV) from Egypt (Eg); Milk vetch dwarf virus (MDV) from Japan (Jp); and Subterranean clover stunt virus (SCSV) and Banana bunchy top virus (BBTV) from Australia (Au).

\begin{tabular}{|c|c|c|c|c|c|c|c|c|}
\hline A) CR-SL & & a & stem & loop & & stem & & \\
\hline DNA-M & $935-$ & ATGACGTCATTTGATCCTTTG TGA & . CTGGGGCGGG & СтTAGTATT & & CAG & ACT & -28 \\
\hline DNA-U2 & $950-$ & AIGACGTCATTTGATCCTTTGOTGA & . CTGGGGCGGGG & СTTAGTATT & AC & $\operatorname{CCCCGCCCCAG}$ & GCPCACGGGATCACT & -28 \\
\hline DNA-U4 & $927-$ & GIGACGTCATTTGATCCTTTGdTGAG & . CTGGGGCGGGG & СTTAGTATT & AC & $\operatorname{ccccGC}$ & GCFCACGGGATCACT & -28 \\
\hline DNA-C & $950-$ & CIGACGTCATTTGATCCTATGATGAG & . СTGGGGCGGGG & СтTAGTATT & $\mathrm{AC}$ & $\mathrm{CCCCGCCCCAG}$ & GTPCACGGGATCACT & -28 \\
\hline DNA-R & $957-$ & ATGACGTCACTTGATCCTATGATGAG & . CTGGGGCGGGG & СTTAGTATT & AC & $\operatorname{CCCCGCCCCAG}$ & GTFCACGGGATCACT & -28 \\
\hline DNA-UI & $945-$ & GLGATMCATTTGATCCTATGATGAG & . CTGGGGCGGGG & CTTAGTATT & AC & CCCCGCCCCAG & GTPCACGGGATCACT & -28 \\
\hline DNA-N & $955-$ & GGGACACTGTTTGATCCTTGG. ACAG & ССTGGCACGGGG & CTTAGTATT & AC & CGTGCCAGG & TTCAAGGGATCAAA & -28 \\
\hline DNA-S & $956-$ & GGAATACTGTTTGATCCTGGG. ACAG & ССTGGCACGGGG & СTTAGTATT & $\mathrm{AC}$ & CGTGCCAGG & ACT & -28 \\
\hline
\end{tabular}

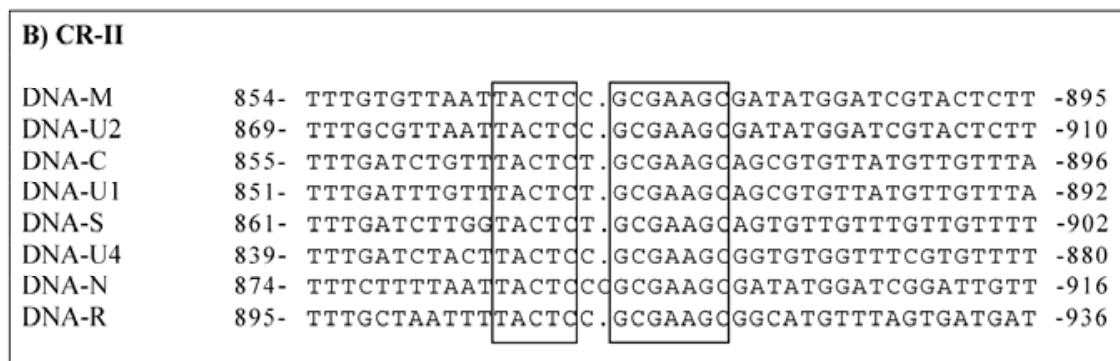

Fig. 3. A, Alignment of the sequences of the origin of replication of the eight DNAs of the nanovirus isolate Eth-231 illustrating the extent of sequence conservation in the stem-loop common region (CR-SL). Inverted repeat sequences (open horizontal arrows) potentially forming a stem-loop structure are in bold. A vertical arrow indicates the "origin of replication", which is the position of cleavage by the master Rep protein Conserved iteron-like sequences (a) and their respective inversions (a') possibly acting as recognition or binding sites for the master replication protein are boxed and indicated by horizontal solid arrows. Dots indicate gaps included to maximize alignments. The Aatl site (GACGTC) conserved in many nanoviruses but absent from DNA- U1, -N, and -S of Eth-231 is shown in bold italics. B, Alignment in the common region II (CR II) showing the perfectly conserved domains (boxed) where endogenous primers may initiate the self priming of genomic DNAs. 
signal (AATAAA) or related sequence required for efficient termination of transcription (29) is appropriately located downstream of the ORFs in all the DNAs.

Comparison of the Eth-231 proteins with those of other nanoviruses. Each of the DNAs has only one ORF potentially encoding a protein ranging in size from 12 to $33 \mathrm{kDa}$ (Table 5). These proteins are homologous to those of other nanoviruses. The amino acid sequence identities resulting from pairwise comparisons of the eight proteins of Eth-231 with those of other nanoviruses are presented in Table 6. When considering all eight proteins, Eth-231 shares an overall protein amino acid sequence identity of $<75 \%$ with other nanoviruses, indicating that Eth-231 is as different from FBNYV and FBNSV as it is from MDV. It is more distantly related to SCSV, with mean amino acid sequence identities of $55 \%$ for the six known homologous proteins, ranging from $37 \%$ for the U1 protein to $82.2 \%$ for the M-Rep protein. The M-Rep protein contains an NTP-binding motif (GGEGKTS) that is conserved in all Rep proteins of nanoviruses (32). With an M-Rep protein amino acid sequence identity ranging from $53.1 \%$ for BBTV to $92.0 \%$ for MDV (Table 6), this protein is the most conserved. On the other hand, the U4 protein is the least conserved when compared with its homologues in FBNYV isolates with identities ranging from 54.7 to $57.1 \%$. In general, the proteins encoded by DNA-U1, -U2, and -U4 whose functions are unknown, are more variable than those encoded by the DNA-R, -N, $-\mathrm{S}$, and $-\mathrm{M}$.

Also, the cell-cycle link (Clink) protein encoded by DNA-C of Eth-231 is unusually divergent, sharing amino acid sequence identities of only 58 to $60 \%$ with the homologous proteins of FBNYV, FBNSV, and MDV. This is partly due to a stretch of 17 amino acids (aa) (positions 101 to 117) which differs strikingly from the corresponding region in the Clink protein of other nanoviruses, although this domain is a fairly conserved Clink domain in members of the genus Nanovirus (Fig. 5). Most notably, the LXCXE motif, which invariably occurs in the Clink protein of all other members of the family Nanoviridae and has been demonstrated to interact with retinoblastoma-like plant proteins for cellcycle regulation $(8,34)$, is absent from the amino acid sequence deduced from the DNA-C sequence of Eth-231.

Phylogenetic analyses of the proteins encoded by each of the four DNAs identified from Eth-218 and by the eight DNAs of Eth-
231 are shown in Figure 2. As expected from the serological data (Table 3) and sequence comparisons (Table 6), Eth-218 formed a tight cluster with FBNYV isolates. When comparing all eight proteins of Eth-231, some of its proteins (i.e., NSP, Clink, and M-Rep) appeared to be phylogenetically intermediate between SCSV and the other known nanoviruses FBNYV, FBNSV, and MDV.

\section{Discussion}

The serological and sequence data presented here indicate that serogroup A isolates very similar to the previously characterized FBNSV isolate from Holetta, Ethiopia $(18,19)$ are the most prevalent in Ethiopia. Therefore, the nanovirus isolates studied in earlier serological surveys and referred to as FBNYV $(3,31)$ may have actually been isolates of FBNSV. This notion is supported by the fact that all the Ethiopian nanovirus isolates studied by Franz et al. (15) had an epitope profile similar to that of FBNSV. Nevertheless, nanoviruses infecting faba bean in the country are highly diverse. Molecular evidence for the occurrence of FBNYV sensu stricto represented by the Ethiopian serogroup B isolates is presented here for the first time. In addition, serogroup $\mathrm{C}$ isolates have not been reported previously. The occurrence of all previously known and new nanovirus isolates together with the report on the occurrence of at least two distinct nanovirus species in Morocco (4) indicates that the previous suggestion about "geographically associated variation of FBNYV isolates" (15) appears to be a result of limited sampling. Together with the results from a previous study $(4,15)$, our data suggest that, in contrast to all other African countries for which information is available, FBNSV is the predominant nanovirus infecting faba bean in Ethiopia. In contrast to FBNSV, FBNYV (serogroup B) and serogroup $C$ isolates were found in only a limited number of samples and very confined geographical areas in southern Ethiopia, where faba bean or legumes in general are grown only at a limited scale. It is not clear why only serogroup A (FBNSV) isolates occur in the main faba-bean-growing areas while a diverse group of nanoviruses occur in the restricted area Gedeo, where faba bean is a minor crop.

Distinction between viruses or their strains is critical in epidemiological surveys because the isolates may differ in their pathogenicity, host range, vector specificity, and geographical distribution. However, only a few studies have been carried out on the

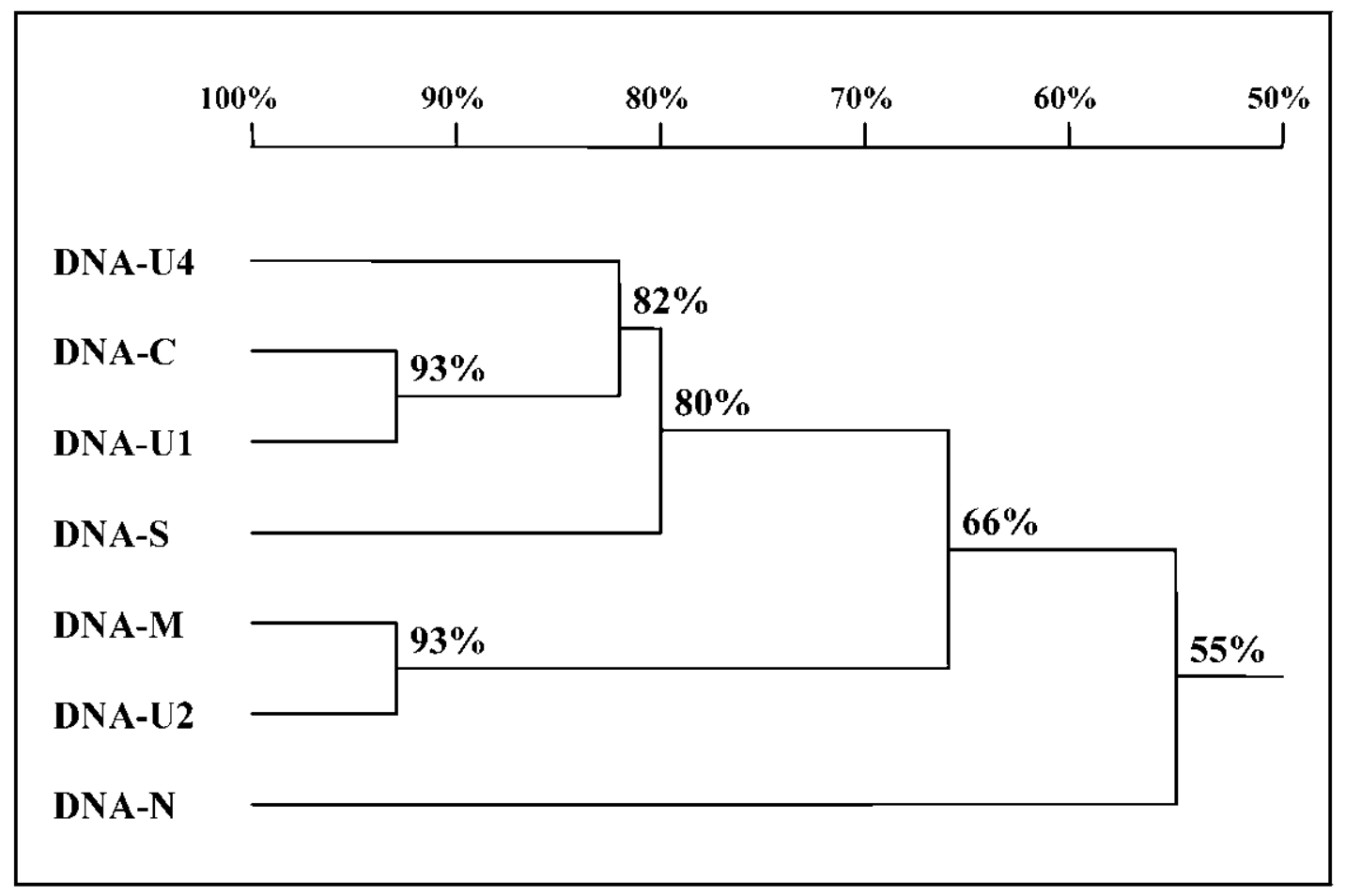

Fig. 4. Identity tree illustrating the relationship among the nucleotide sequences of the noncoding regions of the seven non-Rep DNAs of Eth-231. 


\begin{tabular}{|c|c|c|c|}
\hline Eth-231 & 1 & LREKIVREHMKEERKKEFLENS IEDSCRRYEALLNEDPSSVN . LRK & 57 \\
\hline FBNYV & 1 & ---KA-------HVS--KS---PSE.MYS & 57 \\
\hline FBNSV & 1 & -D-LR---------A----- - HQS-IT--- - TED. -LS & 57 \\
\hline MDV & 1 & $-----\mathrm{KA}------\mathrm{H}----\mathrm{I}----\mathrm{PAE} .-\mathrm{NS}$ & 57 \\
\hline SCSV & 1 & MN - -L- - IK- - - - - - V-KAA-AVF-G-TKKESVEEDDILR & 58 \\
\hline Eth-231 & 58 & LSNYLDLLADYVGNQFNRRCLIRWKKDVPCRVKYGVIEEEHMKLSAYLHLVDFDYGEL & 115 \\
\hline FBNYV & 58 & $--\mathrm{KF}-\mathrm{S}-\mathrm{S}----\mathrm{R}-\mathrm{-}-\mathrm{T}-\mathrm{-}-\mathrm{K}-\mathrm{R}----\mathrm{ANI}-\mathrm{FQ}-\mathrm{M}-\mathrm{-Q}-\mathrm{LR}-\mathrm{YGF}-\mathrm{DMD}-\mathrm{L}$ & 113 \\
\hline FBNSV & 58 & -CKF-ES--Y-------T----K-R----FQI----M--Q-I - - YGI - DME-I & 113 \\
\hline MDV & 58 & 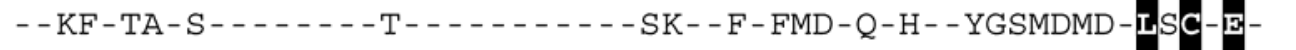 & 113 \\
\hline SCSV & 59 & F-GF-EG-SA-YAEATKKK- -V----S-AINL-WR-M- -M-Y- - YGFADME-LYCSE- & 115 \\
\hline Eth-231 & 116 & FPSL LPLEEDDDVSYVDGTIVRCSLLDFVHSQLDINVVYITVSKNRICTPLRKNCNLYL & 174 \\
\hline FBNYV & 114 & L- . . . P----IT-E--M--N--E--KLFAA-G-R-----N-C----N-DIVIS & 169 \\
\hline FBNSV & 114 & LI . . . P--E--IT-E--I--N-KQ- -NLFAE-G----------C-H---N-EIVIN & 169 \\
\hline MDV & 114 & -I . . . PD-E--LT-E--V-----Q--QLFKS-G-EI---V---HC-WA--S-EIVIK & 169 \\
\hline SCSV & 116 & GFP . . NYGE---A-H- -A--N-KQ-EV-FDD-G-EFMSIVIDR . . . . . . GSIKIE- & 164 \\
\hline
\end{tabular}

Fig. 5. Alignment of the predicted amino acid sequence of the cell-cycle link protein of Eth-231 with those of members of the genus Nanovirus. Note that the protein has a conserved F-box like domain at the $5^{\prime}$ end (highlighted and boxed) but the LXCXE motif (highlighted) conserved in all known nanoviruses is not present in the corresponding region of Eth-231. A possible alternative motif (LXLXE) is boxed. Amino acid residues identical to those of the Eth-231 protein are indicated by a dash, and single dots denote gaps. FBNYV = Faba bean necrotic yellows virus, FBNSV = Faba bean necrotic stunt virus, MDV = Milk vetch dwarf virus, and SCSV = Subterranean clover stunt virus.

variability of nanoviruses worldwide. Karan et al. (23) and Bell (10) studied the genetic variability of the DNA-R of BBTV isolates from different countries and different parts of Vietnam, respectively. Such information has been lacking for legume nanoviruses. Together with recent data on Moroccan nanovirus isolates (4), this study provides useful information on the diversity and coexistence of legume nanoviruses within a country. Hence, it is likely that adequate sampling and analysis of isolates in countries with significant legume production can reveal the coexistence of known or new nanoviruses.

Our results also indicate that there is a good correlation between serotyping using differentiating MAbs and differences in CP and $\mathrm{U} 1$ gene sequences, indicating that the two techniques are complementary. Similar conclusions were reached after detailed comparison of serological and molecular techniques for typing of RNA plant viruses such as Rice yellow mottle virus (14) and Plum pox virus (12). Therefore, large-scale serological analysis of samples from a region followed by sequencing of one or more genes of isolates representing different serogroups as done in our work would be a better approach to understand the diversity or variability of legume nanoviruses in a country or a region.

Like the other previously sequenced isolates of FBNYV, FBNSV, and MDV, eight distinct circular ssDNAs were found associated with Eth-231 infection. Because these DNAs are homologous to that of FBNSV for which infection was demonstrated using cloned DNAs (19) and suggested for other nanoviruses (33), we strongly believe that these eight DNAs comprise the entire genome of Eth-231.

In addition to being genetically distinct from other nanoviruses, the Eth-231 genome had two noteworthy features. (i) In contrast to all other DNA-U4 sequences that have two possible initiation (ATG) codons in close proximity to each other $(3,18,19$; GenBank accession number AB255373), the DNA-U4 of Eth-231 lacks the second ATG, suggesting that DNA-U4 of not only Eth-231 but also other nanoviruses encodes a $12-\mathrm{kDa}$ rather than a $10-\mathrm{kDa}$ protein. (ii) Sequencing of five clones confirmed that DNA-C of Eth-231 encodes a Clink protein that lacks a typical LXCXE motif. This surprising observation suggests that the LXCXE motif is replaced by an FXYXE motif in the Eth-231 Clink protein or that the latter uses an LXLXE motif 6 aa downstream of the FXYXE motif (Fig. 5). One also cannot rule out the possibility that, as has been suggested for begomoviruses (7), this virus may use a quite different motif to interact with retinoblastoma-like proteins.

Taken together, the results revealed that, in addition to FBNSV as the predominant faba-bean-infecting nanovirus in Ethiopia, FBNYV occurs as a second nanovirus on this host in Ethiopia. Furthermore, Eth-231, a previously unrecognized nanovirus species, is serologically distinct and shares overall nucleotide sequence identities of only 70 to $73 \%$ with other nanoviruses. This is clearly below the nucleotide sequence identity threshold of $75 \%$ currently used as a major criterion for nanovirus species demarcation by ICTV (33). In addition, Eth-231 differs considerably in its noncoding sequence from other nanoviruses (Fig. 3) and is also unique in lacking an LXCXE motif in its Clink protein. Although Eth-231 shares CP amino acid sequence identities of 87.2 and 88.4\% with FBNSV and FBNYV, respectively (Table 6) and, thus, does not fully meet another molecular criterion for nanovirus species demarcation (differences of $>15 \%$ in CP amino acid sequences; 33), all other data presented here provide compelling evidence for considering Eth-231 a distinct nanovirus species. The plant from which Eth-231 was identified showed a striking leaf yellowing; therefore, the name faba bean yellow leaf virus (FBYLV) is proposed for this hitherto undescribed nanovirus. Therefore, three distinct nanoviruses, FBNYV, FBNSV, and FBYLV, occur in Ethiopia. Further data on biological properties such as symptom variation, host range, and aphid vectors would be desirable prior to considering FBYLV as a definitive member of the genus Nanovirus.

\section{Literature Cited}

1. Abraham, A., and Albrechtsen, S. E. 1998. Seed-borne viruses infecting some important crops in Ethiopia. Pest Manage. J. Ethiopia 2:94-101.

2. Abraham, A., and Makkouk, K. M. 2002. The incidence and distribution of seed-transmitted viruses in pea and lentil seed lots in Ethiopia. Seed Sci. Technol. 30:567-574.

3. Abraham, A., Makkouk, K. M., Gorfu, D., Lencho, A. G., Ali, K., Tadesse, N., Yusuf, A., and Lencho, A. 2000. Survey of faba bean (Vicia faba L.) virus diseases in Ethiopia. Phytopathol. Mediterr. 39:277-282.

4. Abraham, A. D., Bencharki, B., Torok, V. A., Katul, L. Varrelmann, M., and Vetten, H. J. 2010. Two distinct nanovirus species infecting faba bean in Morocco. Arch. Virol. 155:37-46, 1921-1922 (Erratum).

5. Abraham, A. D., Menzel, W., Lesemann, D.-E., Varrelmann, M., and Vetten, H. J. 2006. Chickpea chlorotic stunt virus: a new polerovirus infecting coolseason food legumes in Ethiopia. Phytopathology 96:437-446.

6. Altschul, S. F., Madden, T. L., Schaffer, A. A., Zhang, Z. H., Zhang, Z., 
Miller, W. W., and Lippman, D. J. 1997. Gapped BLAST and PSI-BLAST: a new generation of protein database search programs. Nucleic Acids Res. 25:3389-3402.

7. Arguello-Astorga, G., Lopez-Ochoa, L., Kong, L.-J., Orozco, B. M., Settlage, S. B., and Hanley-Bowdoin, L. 2004. A novel motif in geminivirus replication protein interacts with the plant retinoblastoma-like protein. $\mathrm{J}$. Virol. 78:4817-4826.

8. Aronson, M. N., Meyer, A. D., Gyorgyey, J., Katul, L., Vetten, H. J., Gronenborn, B., and Timchenko, T. 2000. Clink, a nanovirus encoded-protein binds both pRB and SKP1. J. Virol. 74:2967-2972.

9. Bekele, B., Kumari, S. G., Ali, K., Yusuf, A., Makkouk, K. M., Aslake, M., Ayalew, M., Girma, G., and Hailu, G. 2005. Survey of viruses affecting legume crops in Amhara and Oromia Regions of Ethiopia. Phytopathol. Mediterr. 44:235-246.

10. Bell, K. E., Dale, J. L., Ha, C. V., Vu, M. T., and Revill, P. A. 2002. Characterization of Rep-encoding components associated with banana bunchy top nanovirus in Vietnam. Arch. Virol. 147:695-707.

11. Burns, T. M., Harding, R. M., and Dale, J. L. 1995. The genome organization of banana bunchy top virus: analysis of six ssDNA components. J. Gen. Virol. 76:1471-1482.

12. Candresse, T., Cambra, M., Dallot, S., Lanneau, M., Asensio, M., Gorris, M., Revers, F., Macquaire, G., Olmos, A., Quiot, J.-B., and Dunez, J. 1998. Comparison of monoclonal antibodies and polymerase chain reaction assays for the typing of isolates belonging to the $\mathrm{D}$ and $\mathrm{M}$ serotypes of plum pox potyvirus. Phytopathology $88: 198-204$.

13. FAOSTAT. 2008. http://apps.fao.org/faostat

14. Fargette, D., Pinel, A., Halimi, H., Brugidou, C., Fauquet, C., and van Regenmortel, M. H. V. 2002. Comparison of molecular and immunological typing of isolates of Rice yellow mottle virus. Arch. Virol. 147:583-596.

15. Franz, A., Makkouk, K. M., Katul, L., and Vetten, H. J. 1996. Monoclonal antibodies for the detection and differentiation of faba bean necrotic yellows virus isolates. Ann. Appl. Biol. 128:255-2268.

16. Franz, A., Makkouk, K. M., and Vetten, H. J. 1997. Host range of faba bean necrotic yellows virus and potential yield loss in infected faba bean. Phytopathol. Mediterr. 36:94-103.

17. Franz, A., Makkouk, K. M., and Vetten, H. J. 1998. Acquisition, retention and transmission of faba bean necrotic yellows virus by two of its aphid vectors, Aphis craccivora Koch and Acyrthosiphon pisum Harris. J. Phytopathol. 146:347-355.

18. Franz, A., van der Wilk, F., Verbeek, M., Dullemans, A. M., and van den Heuvel, J. F. J. M. 1999. Faba bean necrotic yellows virus (Genus $\mathrm{Na}$ novirus) requires a helper factor for its aphid transmission. Virology 262:219-219.

19. Grigoras, I., Timchenko, T., Katul, L., Grande-Perez, A., Vetten, H. J., and Gronenborn, B. 2009. Reconstitution of authentic nanovirus from multiple cloned DNAs. J. Virol. 83:10778-10787.

20. Hafner, G. J., Harding, R. M., and Dale, J. L. 1997. A DNA primer associ- ated with banana bunchy top virus. J. Gen. Virol. 78:479-486.

21. Hughes, A. L. 2004. Birth-and-death evolution of protein coding regions and concerted evolution of non-coding regions in the multi-component genomes of nanoviruses. Mol. Phylogenet. Evol. 30:287-294.

22. International Committee on Taxonomy of Viruses, 2010. http://talk ictvonline.org/files/proposals/taxonomy_proposals_plant1/m/plant04/3840. aspx

23. Karan, M., Harding, M. R., and Dale, J. L. 1994. Evidence for two groups of banana bunchy top virus isolates. J. Gen. Virol. 75:3541-3546.

24. Katul, L., Maiss, E., Morozov, S. Y., and Vetten, H. J. 1997. Analysis of six DNA components of the faba bean necrotic yellows virus genome and their structural affinity to related plant virus genomes. Virology 233:247-259.

25. Katul, L., Timchenko, T., Gronenborn, B., and Vetten, H. J. 1998. Ten distinct ssDNA components, four of which encode putative replicase-associated proteins, are associated with the faba bean necrotic yellows virus genome. J. Gen. Virol. 79:3101-3109.

26. Katul, L., Vetten, H. J., Maiss, E., Makkouk, K. M., Lesemann. D.-E., and Casper, R. 1993. Characterization and serology of virus-like particles associated with faba bean necrotic yellows. Ann. Appl. Biol. 123:629-647.

27. Makkouk, K. M., and Kumari, S. G. 2009. Epidemiology and integrated management of persistently transmitted aphid-borne viruses of legume and cereal crops in West Asia and North Africa. Virus Res. 141:209-218.

28. Makkouk, K. M., Vetten, H. J., Katul, L., Franz, A., and Madkour, M. A. 1998. Epidemiology and control of faba bean necrotic yellows virus. Pages 534-540 in: Plant Virus Disease Control. A. Hadidi, R. K. Khetarpal, and H. Koganezawa, eds. American Phytopathological Society, St. Paul, MN.

29. Rothnie, H. M., Reid, J., and Hohn, T. 1994. The contribution of AAUAAA and the upstream element UUUGUA to the efficiency of mRNA 3' end formation in plants. EMBO J. 13:2200-2210.

30. Sano, Y., Wada, M., Hashimoto, Y., Matsumoto, T., and Kojima, M. 1998 Sequence of ten circular ssDNA components associated with the milk vetch dwarf virus genome. J. Gen. Virol. 79:3111-3118.

31. Tadesse, N., Ali, K., Gorfu, D., Yusuf, A., Abraham, A., Ayalew, M., Lencho, A., Makkouk, K. M., and Kumari, S. G. 1999. Survey of chickpea and lentil virus diseases in Ethiopia. Phytopathol. Mediterr 38:149-158.

32. Timchenko, T., de Kouchkovsky, F., Katul, L., David, C., Vetten, H. J., and Gronenborn, B. 1999. A single rep protein initiates replication of multiple genome components of faba bean necrotic yellows virus, a single stranded DNA virus of plants. J. Virol. 73:10173-10182.

33. Vetten, H. J., Dale, J. L., Grigoras, I, Gronenborn, B., Harding, R., Randles, J. W., Sano, Y., Thomas, J. E., Timchenko, T., and Yeh, H.-H. 2011. Family Nanoviridae. Pages 395-404 in: Virus Taxonomy, Ninth Report of the International Committee on Taxonomy of Viruses. A. M. Q. King, M. J. Adams, E. C. Carstens, and E. J. Lefkowitz, eds. Elsevier/Academic Press, London.

34. Wanitchakorn, R., Hafner, G. J., Harding, R. M., and Dale, J. L. 2000. Functional analysis of proteins encoded by banana bunchy top virus DNA-4 to -6 . J. Gen. Virol. 81:299-306. 